\title{
Universal Proximity Effect in Target Search Kinetics in the Few-Encounter Limit
}

\author{
Aljaž Godec ${ }^{1,2, *}$ and Ralf Metzler ${ }^{1, \dagger}$ \\ ${ }^{1}$ Institute of Physics \& Astronomy, University of Potsdam, 14476 Potsdam-Golm, Germany \\ ${ }^{2}$ Department of Molecular Modeling, National Institute of Chemistry, 1000 Ljubljana, Slovenia \\ (Received 17 July 2016; revised manuscript received 7 September 2016; published 21 November 2016) \\ When does a diffusing particle reach its target for the first time? This first-passage time (FPT) problem is \\ central to the kinetics of molecular reactions in chemistry and molecular biology. Here, we explain the \\ behavior of smooth FPT densities, for which all moments are finite, and demonstrate universal yet \\ generally non-Poissonian long-time asymptotics for a broad variety of transport processes. While Poisson- \\ like asymptotics arise generically in the presence of an effective repulsion in the immediate vicinity of the \\ target, a time-scale separation between direct and reflected indirect trajectories gives rise to a universal \\ proximity effect: Direct paths, heading more or less straight from the point of release to the target, become \\ typical and focused, with a narrow spread of the corresponding first-passage times. Conversely, statistically \\ dominant indirect paths exploring the entire system tend to be massively dissimilar. The initial distance to \\ the target particularly impacts gene regulatory or competitive stochastic processes, for which few binding \\ events often determine the regulatory outcome. The proximity effect is independent of details of the \\ transport, highlighting the robust character of the FPT features uncovered here.
}

DOI: 10.1103/PhysRevX.6.041037

\section{INTRODUCTION}

Exactly 100 years ago, Smoluchowski derived the rate for the reactive encounter of two diffusing particles [1]. Based on Smoluchowski's concepts, first-passage time statistics characterize the diffusion limitation of molecular reactions [2-13]. A case of particular relevance is cellular signaling by specific molecules [13-21]. The existence and impact of significant sample-to-sample fluctuations in the precise timing of cellular regulation, often at lowcopy numbers of the signaling molecules, are by now well established both experimentally and theoretically [8,18-20,22-26]. Remarkably, despite the highly heterogeneous character of the motion of signaling molecules in the nucleus and cell cytoplasm [27-29], the overall signaling kinetics appears to be universally stable, thus allowing cellular operation at a remarkable precision [30]. In the particular case of transcription regulation, the experimentally observed strong positional correlations between pairs of downstream coregulated genes in both prokaryotic [21] and eukaryotic cells [31] indicate that proximity may indeed represent a tuning mechanism, which was also supported theoretically in a three-dimensional (3D) setting [15]. According to Ref. [8], such proximity effects should

\footnotetext{
*agodec@uni-potsdam.de

†rmetzler@uni-potsdam.de
}

Published by the American Physical Society under the terms of the Creative Commons Attribution 3.0 License. Further distribution of this work must maintain attribution to the author $(s)$ and the published article's title, journal citation, and DOI.
Subject Areas: Biological Physics, Chemical Physics, Statistical Physics only be relevant for so-called geometry-controlled kinetics when the molecules explore their surrounding space in a compact manner, that is, for recurrent motion [32] such as one-dimensional (1D) diffusion or diffusion on fractals [8]. Currently, there is no consensus on whether the eukaryotic chromosome has a fractal structure [33,34]. In most bacteria, the DNA is segregated and spatially highly organized yet lacks a fractal organization [35]. In this context, the immediate question arises as to whether compact exploration is indeed necessary for the observed regulatory precision $[30,36]$ : namely, are proximity effects truly limited to compact exploration-or could there in fact be a more general mechanism to boost signaling speed and precision?

Beyond the scope of transcription regulation, initial distances in spatial search processes are important in a large variety of contexts, ranging, e.g., from locating enemy vessels in the ocean [37] and search strategies of animals foraging for food [38] to the global spreading of diseases [39]. Here, the initial distance to the target is often essential $[37,40,41]$; in particular, it plays an important role in the foraging behavior of animals when food resources are sparse [38], or in the predation behavior of marine vertebrates, including basking sharks [42], jellyfish, leatherback turtles [43], and southern elephant seals [44]. Proximity becomes essential in the first-come-first-serve sense corresponding to a destructive search limit [40], i.e., when the target (food) disappears upon the first time location by a searcher.

A key experimental factor in gene regulation is the fact that, in each realization, only the fastest few of the $10^{2}$ to 
$10^{4}$ transcription factor molecules relaying the biochemical signal to a gene determine the outcome (i.e., the cell's response) [45]. Similarly, the arrival of the fastest sperm cell at the egg cell decides the future of the new organism to be formed in each particular case. As we demonstrate below, this situation is fundamentally very different from the question of how long it takes for the first signal to arrive, on average, in an ensemble of independent nondestructive search realizations (see, e.g., Refs. [8,46,47]). This average scenario could correspond, e.g., to ensemble experiments of molecular signaling effects in a colony of genetically identical cells. The characteristic arrival time of the fastest searcher in an ensemble of nondestructive search realizations is influenced, to a dominant extent, by massively dissimilar indirect trajectories, i.e., those that interact with the confining boundary before reaching the target $[8,18,25]$. The statistics of such indirect trajectories was found to exhibit a qualitatively different behavior with respect to the compactness of spatial exploration in the limit of a large system size and were therefore suggested to define two different universality classes [8]. How is this finding compatible with the fact that the FPT statistics in both cases follow an exponential asymptotic decay? And moreover, is the long-time behavior of the FPT statistics also sufficient to describe the first-arrival kinetics in each given realization, such as encountered in transcription regulation?

Here, we address these questions in two steps. First, without specifying the actual motion pattern, we prove that the long-time behavior of all molecular target search processes in a confined and, in particular, small domain belongs to the same universality class, irrespective of whether the dynamics is compact or not. Second, we analyze various specific types of motion patterns and show how Poisson-like asymptotics arise if the particle experiences an effective repulsion in the immediate vicinity of the target [Figs. 1(a) and 1(b)]. In contrast, the proximity effects we uncover and quantify here naturally lead to an accumulation of probability for observing direct trajectories for both compact and noncompact Poisson-like dynamics [Fig. 1(c)], which enhances the speed and precision of the target search process. In this sense, to rationalize the proximity effect in gene regulation, one needs to consider explicitly the statistics of direct trajectories, i.e., those that do not interact with the confining boundary before reaching the target. Using experimentally relevant parameters for transcription regulation, we argue that proximity effects may indeed represent a universal means for tuning cellular signaling kinetics.

The paper is structured as follows. First, we represent a general theory for the long-time behavior of smooth densities, for which all moments are finite, and establish a unification of the previously proposed universality classes in the finite volume case. Next, we demonstrate, based on exact result for a variety of different passive and driven (a)

(b) Poissonian asymptotic
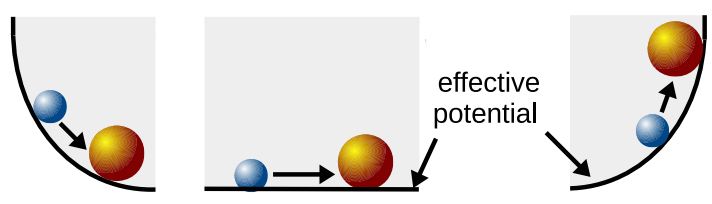

(c)

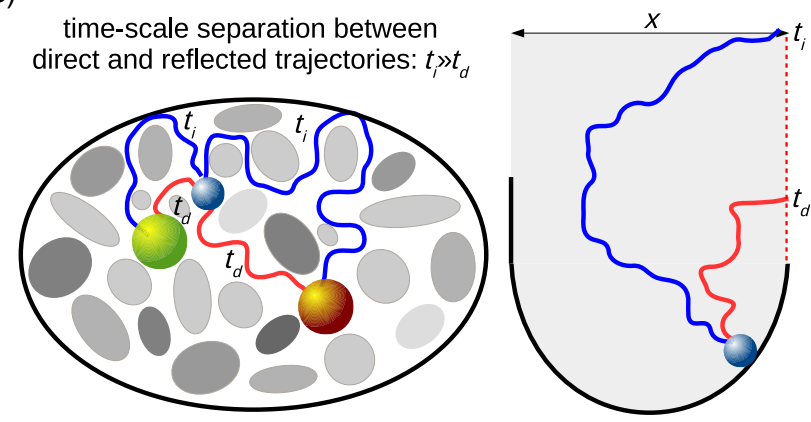

direct trajectories are typical, indirect ones are strongly dissimilar

FIG. 1. Schematic of the two limiting forms of long-time FPT behavior, (a) and (b), depending on the effective potential experienced by the searcher in the vicinity of the absorbing target (originating either from an external force or from the geometric spurious drift in the radial direction). (c) Schematics of two molecules (large spheres) searching for a target (small sphere) with two different realizations of direct and indirect trajectories $t_{d}$ and $t_{i}$, respectively, depending on the initial distance to the target (see text for details). As soon as a timescale separation exists between $t_{d}$ and $t_{i}$, a robust proximity effect emerges, with any two $t_{d}$ being typical and very similar while any two $t_{i}$ tend to be strongly dissimilar. This focusing of $t_{d}$ in turn enables faster and more precise gene regulation.

diffusion processes, the existence of a universal proximity effect in the presence of a time-scale separation between direct and indirect trajectories. Then, we discuss the biophysical implications of these results in the context of transcription regulation. We conclude with a summary of our results and offer a perspective on the importance of our results for future studies.

\section{THEORY AND GENERAL RESULTS FOR THE LONG-TIME BEHAVIOR}

To address the problem in the most general setting, we impose only mild constraints, which are warranted by the physical setting: We assume the finiteness of the moments $\left\langle T^{n}\right\rangle$ of the FPT density. This means that the dynamics has a finite natural time scale, as expected in a finite volume. Then, the Laplace transform of the FPT density, $\tilde{\wp}(s)=\hat{\mathcal{L}}[\wp: t \rightarrow s]$, of a particle starting at position $x_{0}$ to arrive at the target at $x_{a}$ (Fig. S1), admits a moment expansion $\tilde{\wp}(s)=\sum_{i=0}^{\infty}\left\langle T^{n}\right\rangle(-s)^{n} / n !$. Moreover, it has the following representation in the form of a quotient of convergent power series: 


$$
\tilde{\wp}(s)=\frac{g\left(s ; x_{0}\right)}{h\left(s ; x_{a}\right)} \equiv \frac{\sum_{k=0}^{\infty} g^{(k)}\left(x_{0}\right) s^{k} / k !}{\sum_{k=0}^{\infty} h^{(k)}\left(x_{a}\right) s^{k} / k !}
$$

(see Eq. (S1) in Ref. [48]), where the superscript $(k)$ denotes the $k$ th derivative with respect to $s$ evaluated at $s=0$ [49]. Here, we limit the discussion to hyperspherically symmetric Markov systems, but our general result can be readily applied to any geometry given the knowledge of $g^{(k)}, h^{(k)}$. By Cauchy's theorem, the asymptotic behavior of $\wp(t)$ is determined by the root of $h(s)$ that is closest to the origin at $s=0$.

In a confined domain, the transport operator $\hat{L}$ for such dynamics has a discrete, real, nondegenerate eigenvalue spectrum $\left\{\lambda_{i}\right\}$ with eigenvectors $\psi_{i}$. Moreover, $\lambda_{i}<0$ for all $i$ because of the absorbing boundary [50]. The long-time behavior of $\wp(t)$ is determined in terms of the lowest eigenpair $\left(\lambda_{0}, \psi_{0}\right): \wp(t) \sim-\mathcal{K} \psi_{0}\left(x_{0}\right)\left[\partial \psi_{0}\left(x_{a}\right) / \partial x\right] \mathrm{e}^{-\lambda_{0} t}$, where the constant $\mathcal{K}$ takes into account the specific properties of the given system and $\sim$ denotes asymptotic equality.

By necessity, $\lambda_{0}$ is a simple pole. To find it, we must invert $h(s)$ in the vicinity of 0 for negative real $s$. As long as $\tilde{\wp}(s)$ does not have a branch cut along the negative real axis-an assumption that is confirmed below-this can be done exactly in the form of a Newton series (for details, see Ref. [48]). The first step of this procedure consists of obtaining the solution $-\lambda_{0}$ of the nonlinear equation $h\left(s ; x_{a}\right)=0$. The exact result is given by the Newton series (see Ref. [48])

$$
\lambda_{0}\left(x_{a}\right)=\sum_{k=1}^{\infty} \frac{h^{(0)}\left(x_{a}\right)^{k}}{h^{(1)}\left(x_{a}\right)^{2 k-1}} \frac{\operatorname{det} \mathcal{A}_{k}}{(k-1) !}
$$

where $\mathcal{A}_{k}$ is an almost triangular square matrix with elements

$$
\begin{aligned}
\mathcal{A}_{n}(i, j)= & \frac{h^{(i-j+2)} \Theta(i-j+1)}{(i-j+2) !} \\
& \times[n(i-j+1) \Theta(j-2)+i \Theta(1-j)+j-1] .
\end{aligned}
$$

Here, $\Theta(n)$ is the discrete Heaviside step function, and we use the symbolic convention $\operatorname{det} \mathcal{A}_{1} \equiv 1$ [see also Ref. [48], Eq. (S11)]. The series (2) is generally rapidly converging; e.g., for one-dimensional Brownian motion, it converges to within $99.4 \%$ of the known result $\pi^{2} / 4[3,25]$ already at $k=4$. As long as the particle is not strongly biased towards the target, in which case $k \sim \mathcal{O}(100)$, as $\wp(t)$ tends to a delta function, $\lesssim 10$ terms are typically sufficient to achieve convergence to numerical precision. Explicitly, the lowestorder terms of Eq. (2) have the form

$$
\lambda_{0}\left(x_{a}\right) \sim \frac{h^{(0)}\left(x_{a}\right)}{h^{(1)}\left(x_{a}\right)}\left(1+\frac{h^{(0)}\left(x_{a}\right)}{2} \frac{h^{(2)}\left(x_{a}\right)}{h^{(1)}\left(x_{a}\right)^{2}}[1+\mathcal{O}]\right),
$$

where the first terms of the remainder $\mathcal{O}$ read

$$
\begin{aligned}
\mathcal{O}= & \frac{h^{(0)} h^{(2)}}{\left[h^{(1)}\right]^{2}}\left(1+\frac{5}{4} h^{(0)} h^{(2)}\right)-\frac{1}{3} \frac{h^{(0)} h^{(3)}}{h^{(1)} h^{(2)}}\left(1+\frac{5}{2} \frac{h^{(0)} h^{(2)}}{\left[h^{(1)}\right]^{2}}\right) \\
& +\frac{1}{12} \frac{\left[h^{(0)}\right]^{2} h^{(4)}}{\left[h^{(1)}\right]^{2} h^{(2)}}+\cdots
\end{aligned}
$$

After obtaining $\lambda_{0}$, we can isolate the leadingorder term of $\tilde{\wp}(s)$ under the quite loose condition $\lim _{n \rightarrow \infty}\left(g^{(n)} / h^{(n)}\right)<\infty$, which is fulfilled in all the cases studied here and appears to follow generally from the positivity and finiteness of $\left\langle T^{n}\right\rangle$ (for a detailed discussion and justification, see Ref. [48]). Now, it is essentially straightforward to invert the Laplace transform using Cauchy's theorem to obtain the exact long-time behavior of the FPT density (for a detailed proof, see Ref. [48]). The result reads

$\wp(t) \sim \lim _{k \rightarrow \infty} \frac{\sum_{l=0}^{k-1} \frac{1}{l !}\left[g^{(l)}-h^{(l)} \frac{g^{(k)}}{h^{(k)}}\right]\left(-\lambda_{0}\right)^{l}}{\sum_{l=0}^{k-1} \sum_{m=1}^{k-l} \frac{1}{(l+m) !} h^{(l+m)}\left(-\lambda_{0}\right)^{l+m-1}} \mathrm{e}^{-\lambda_{0} t}$.

Note that the moments $\left\langle T^{n}\right\rangle$ can be obtained recursively from the coefficients $g^{(k)}$ and $h^{(k)}$ and vice versa [see Eq. (S2) in Ref. [48] for details]. Equations (2)-(6) give our first main result. These expressions are important as they enable us to obtain the exact long-time behavior of any smooth density that has an exponential asymptotic decay. Equations (2) and (6) are universal in the sense that the obtained asymptotic exponential decay holds for any firstpassage process with a smooth density, for which all $\left\langle T^{n}\right\rangle<\infty$. Note that they also hold irrespective of the initial distance to the target. Namely, while, in general, the Laplace transform of some arbitrary asymptotically exponential density $\tilde{\wp}(s)$ may display multiscaling with respect to $s$ (see, e.g., Ref. [51]), the smoothness and finiteness of $\left\langle T^{n}\right\rangle$ assure exactly the validity of the power series in Eq. (S1), at least in the domain $-\lambda_{0}-\delta<\operatorname{Re}(s)<\lambda_{0}$ for some real $\delta>0$ [51]. This satisfies the requirements for the above result to apply. Our general result should therefore also apply to non-Markovian dynamics as soon as all moments of the FPT density are finite. However, while results for the mean FPT for a broad class of Gaussian non-Markovian processes in bounded domains were obtained recently in Ref. [52] and it is also known that a specific class of confined Gaussian non-Markovian processes indeed has exponential long-time asymptotics [53], the properties of higher moments and the long-time asymptotics of FPT density for a general nonMarkovian process remain elusive.

\section{A. Unification of universality classes}

To first demonstrate the universality implied by Eq. (6), we analyze the FPT behavior in a broad variety of spatially confined systems, in which the stochastic dynamics ranges from compact to noncompact cases, and we also consider 
diffusion in external fields. Specifically, we study unbiased diffusion in hyperspherical domains in the presence of a centered absorbing target in dimensions $1 \leq d \leq 3$. As an extension to Brownian motion, we also examine diffusion in disordered media and fractals with fractal dimension $d_{f}$ $[8,54,55]$. Thus, we analyze the scaling limit of a Markovian random walk, whose mean-squared displacement scales as $\left\langle r^{2}(t)\right\rangle \propto t^{2 / d_{w}}$, where $d_{w} \geq 2$ denotes the random walk dimension and $d_{w}>2$ describes subdiffusive motion [55]. The various different regimes of the FPT behavior are captured by the parameter $\nu=\frac{3}{2}-\left[\left(d_{f}+1\right) / d_{w}\right]$. Among biased diffusion processes, we analyze one-dimensional diffusion in a linear potential (Taylor dispersion) [3], two-dimensional (2D) diffusion in a radial potential flow $[3,56]$. and the Kramer's escape of an overdamped particle from a harmonic potential [2,57-61].

Particle-based computer simulations are carried out to corroborate the analytical results. Passive diffusion and two-dimensional radially biased diffusion are simulated in terms of the (squared) Bessel process with parameter $\nu$ by numerically integrating the Itô stochastic equation $d Y_{t}=$ $(1-2 \nu) / Y_{t}+\eta_{t}$ with zero-mean Gaussian white noise of variance $\left\langle\eta(t) \eta\left(t^{\prime}\right)\right\rangle=2 D \delta\left(t-t^{\prime}\right)$ up to the FPT $Y_{t}=r_{a}$ [62]. Biased one-dimensional diffusion and the Kramer's escape from a parabolic potential are simulated by numerically integrating the Itô stochastic equations: $d Y_{t}=v+\eta_{t}$, and $d Y_{t}=-m \gamma^{-1} \omega^{2} Y_{t}+\eta_{t}^{\prime}$, respectively. Note that $1-5 \times$ $10^{4}$ realizations were taken to measure $\wp(t)$.

According to Eq. (6), the FPT densities for these fundamentally different processes should collapse, after rescaling, to the asymptotic unit exponential $\wp\left(\theta=t / \lambda_{0}\right) /$ $\mathcal{C} \sim \mathrm{e}^{-\theta}$, where $\mathcal{C}$ represents the prefactor in Eq. (6). In excellent agreement, Fig. 2 demonstrates that the rescaled FPT densities indeed collapse to a master curve at long times for all processes considered here. Note the excellent agreement of the data with the predicted single exponential. Therefore, the long-time behavior of all first-passage processes with finite moments unifies in a single universality class - the family of exponential densities. How can this be reconciled with the two different universality classes obtained in Ref. [8]? The solution of this seeming contradiction lies in the exact way the limits are taken, as we detail now.

In Ref. [8], the authors considered passive Markovian diffusion in a (generally fractal) medium with (fractal) dimension $d_{f}$ in a large volume limit of the confining domain. Since such Markovian diffusion is self-similar in arbitrary dimensions [63], the results are invariant with respect to the exact volume (or equivalently, the radius of the domain $R$ ) if we express time in natural units $\tau=R^{d_{w}} / D_{d_{w}}$, where we defined the (generalized) diffusion coefficient $D_{d_{w}}$. Therefore, we can, without any loss of generality, consider the problem in a domain of unit radius and express spatial coordinates in units of $R$ (see below for details of the transformation). In this invariant setting, the large volume limit in Ref. [8] corresponds to the limit when the dimensionless target size $x_{a}$ tends to be very small.

In a passive fractal medium, the particle experiences the aforementioned effective $d_{f^{-}}$and $d_{w}$-dependent geometric outward radial bias (called the "centrifugal drift" in Ref. [3]). As we will show below, the mathematical origin for the different limiting behaviors with respect to $d_{f}$ and $d_{w}$ found in Ref. [8] is actually rooted in the fact that the limit $x_{a} \rightarrow 0$ corresponds to a singular perturbation in the case of noncompact exploration, but merely to a change of the time unit for compact exploration. Mathematically, the limit of a large volume thus has a different meaning for compact and noncompact exploration.
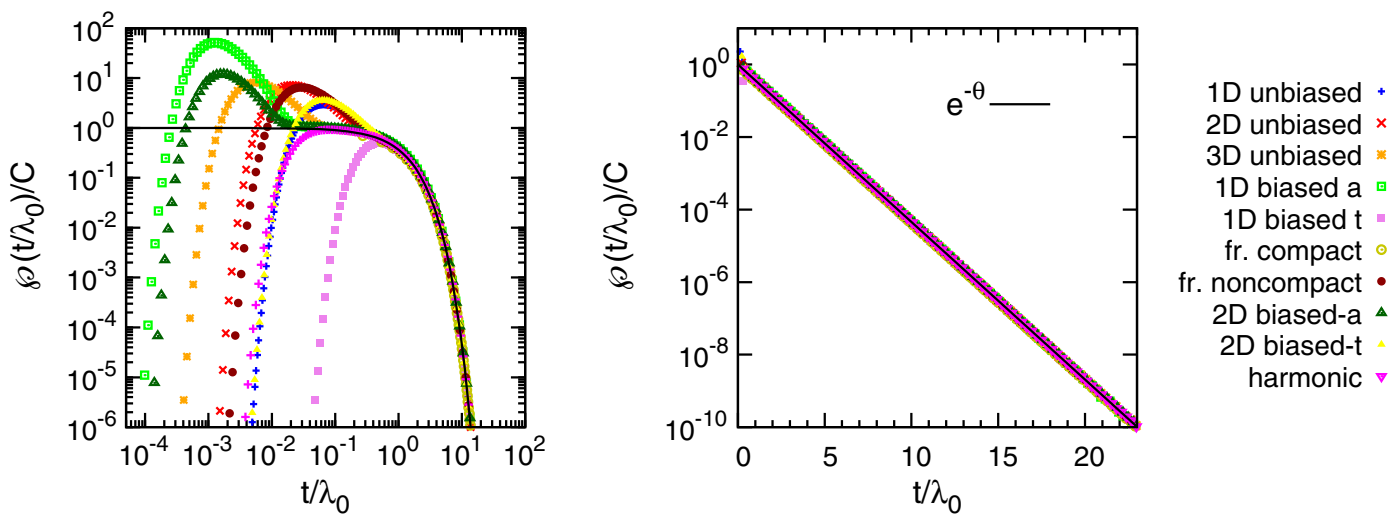

FIG. 2. Asymptotic collapse and short-time nonuniversality for the rescaled FPT densities $\wp\left(\theta=t / \lambda_{0}\right) / \mathcal{C}$ for the various models described in the text. The parameters are $x_{0}=0.4$ (1D diffusion), $x_{a}=0.2, x_{0}=0.5$ ( $2 \mathrm{D}$ diffusion), $x_{a}=0.1, x_{0}=0.4$ ( $3 \mathrm{D}$ diffusion), $x_{0}=0.3, \mathrm{Pe}=3$ (1D biased away from the target), $x_{0}=0.4, \mathrm{Pe}=-3$ (1D biased towards the target), $\nu=-0.25, x_{a}=0.2, x_{0}=0.45$ (noncompact diffusion on fractals), $\nu=0.25, x_{a}=0.2, x_{0}=0.45$ (compact diffusion on fractals), $x_{a}=0.2, x_{0}=0.5, \mathrm{Pe}=3(2 \mathrm{D}$ radial potential flow away from the target), $x_{a}=0.2, x_{0}=0.5, \mathrm{Pe}=-1.8$ ( $2 \mathrm{D}$ radial potential flow towards the target), and $x_{0}=-1$, $x_{a}=2.8$ (overdamped escape from a harmonic potential). 
Another important point in this context follows from the exact limiting behavior of the coefficients $h^{(k)}$ and the rate of convergence of the Newton series (2) when the effective potential experienced by the searching particle in the vicinity of the absorbing target (originating either from an external force or from the geometric spurious drift in the radial direction) becomes sufficiently repulsive, such that, at least locally in the vicinity of the target, the motion is effectively nonrecurrent. As we prove below, in this limit, the first correction $\left[h^{(2)}\left(x_{a}\right) / h^{(1)}\left(x_{a}\right)^{2}\right]$ to the leading-order behavior in Eq. (4) already converges uniformly to 0. Similarly, the prefactor in Eq. (6) converges with the first term, and Eq. (6) reduces to

$$
\wp(t) \sim \frac{\left\langle T\left(x_{0}\right)\right\rangle}{\left\langle T\left(x^{\dagger}\right)\right\rangle} \frac{1}{\left\langle T\left(x^{\dagger}\right)\right\rangle} \mathrm{e}^{-t /\left\langle T\left(x^{\dagger}\right)\right\rangle} .
$$

Here, $\left\langle T\left(x_{0}\right)\right\rangle$ and $\left\langle T\left(x^{\dagger}\right)\right\rangle$ are the mean FPT from the initial location $x_{0}$ and the location $x^{\dagger}$ of the potential minimum. If the potential is monotonic or the bias has a geometric origin, then $x^{\dagger}$ corresponds to the location of the confining boundary.

In the limit $x_{a} \rightarrow 0$, this effective bias becomes sufficiently large for $\frac{3}{2}<\left[\left(d_{f}+1\right) / d_{w}\right] \leq 2$ (i.e., for so-called noncompact spatial exploration [8]) and leads to Eq. (7) the emergence of Poisson-like asymptotics (for details, explicit results, and proofs, see below and Ref. [48]). In contrast, as we show below in the case of compact exploration, i.e., $0<\left[\left(d_{f}+1\right) / d_{w}\right] \leq \frac{3}{2}$, the coefficients $h^{(k)}$ become independent of $x_{a}$ in the limit $x_{a} \rightarrow 0$ as the effective outward bias vanishes. As a result, higher-order terms in Eq. (2) need to be considered as well.

Therefore, the long-time behavior of the FPT density for diffusion in generally fractal media in fact falls into a single universality class; i.e., it can be described by the single universal equation (6). It is only the behavior of the coefficients in Eqs. (2)-(6) that, in the limit of a vanishing target (or large volume, in the language of Ref. [8]), depends on the compactness of the exploration of space. In fact, one can check, by a suitable identification of parameters and a corresponding change of units, that our single universal result reproduces both qualitative regimes of the limiting long-time behavior found in Ref. [8]. In that sense, our results in Eqs. (2)-(6) fully corroborate and unify the findings in Ref. [8]. In addition, here we present the exact forms of the short- and intermediate-time FPT behavior.

We generalize the cases considered in Ref. [8] and demonstrate explicitly that the single universal result also captures diffusion in external fields. In addition, we prove the reduction of Eq. (6) to the Poisson-like behavior (7) for a sufficiently strong locally repulsive potential. An important point we make is that, in all these cases, the long-time behavior is in fact insufficient to describe and explain the kinetics in the few-encounter regime, such as those occurring in gene regulation, and that the cognizance of the full
FPT distribution $\wp(t)$ is needed to quantitatively understand the system.

\section{TIME-SCALE SEPARATION AND THE PROXIMITY EFFECT}

As we will show later, the long-time behavior of $\wp(t)$ is not sufficient to explain the proximity effect. Note that even the emergence of Poisson-like asymptotics in Eq. (7), in general, does not imply the existence of a single time scale in the kinetics. In the presence of a time-scale separation between direct and reflected indirect trajectories [Fig. 1(c)], proximity effects emerge for biased as well as for compact and noncompact passive motion.

In contrast to the long-time asymptotics, the short-time behavior of $\wp(t)$, involving the direct trajectories from the initial position towards the target, which do not feel the presence of the confinement, shows no such universality. While in all the cases studied it contains the Lévy-Smirnov density

$$
\Phi\left(t ; x_{0}-x_{a}\right)=\frac{\left|x_{0}-x_{a}\right|}{2 \sqrt{\pi t^{3}}} \exp \left(-\frac{\left|x_{0}-x_{a}\right|^{2}}{4 t}\right)
$$

describing trajectories propagating directly towards the target, in general this expression is multiplied by a
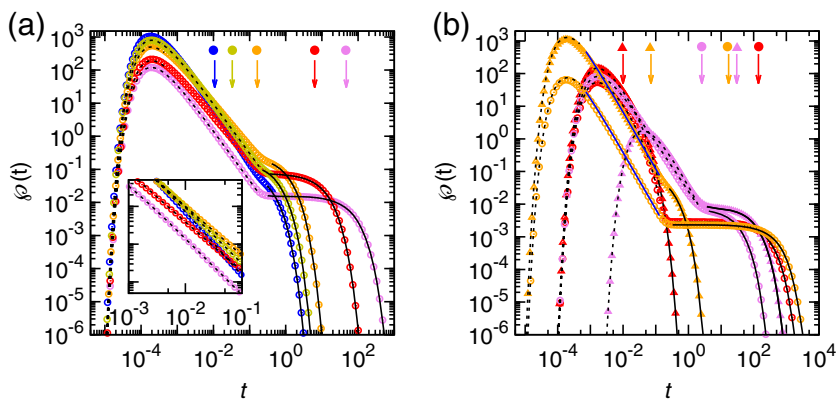

FIG. 3. FPT densities for various passive (a) and biased (b) diffusive systems along with mean FPT values (arrows). (a) $x_{a}=0.006, x_{0}=0.04$ and $\nu=-1 / 2$ (violet), $\nu=-1 / 4$ (red), $\nu=-1 / 4$ (orange), $\nu=1 / 2$ (yellow), and $\nu=0.7$-strong subdiffusion (blue). The symbols represent results of numerical simulations (see Sec. II A), solid lines correspond to Eq. (6) (or the Poissonian limit for $\nu<0$ ), and dashed lines correspond to Eq. (9). Inset: Magnification of the intermediate regime with the asymptotic (10) depicted by dashed lines. Note that the long-time behavior for both $\nu<0$ cases is well within the Poisson-like regime. (b) One-dimensional diffusion under constant bias for $x_{0}-x_{a}=0.1$ [orange; $\mathrm{Pe}=-5$ (circles) and $\mathrm{Pe}=5$ (triangles)], 2D diffusion with radial bias for $x_{a}=0.006, x_{0}=0.04$ [red; $\mathrm{Pe}=0.75$ (circles) and $\mathrm{Pe}=-0.75$ (triangles)], and the OU process [violet; $x_{a}=3, x_{0}=2.5$ (triangles), $x_{a}=2.5$, $x_{0}=2.4$ (circles)]. The symbols represent results of numerical simulations (see Methods), solid lines correspond to Eq. (6) (or the Poissonian limit for $\nu<0$ ), black dashed lines correspond to Eqs. (9) and (15), respectively, and the blue solid lines correspond to Eq. (10). 
nonuniversal prefactor and potentially also a $t$-dependent function (see below). The behavior in Eq. (8) is generally known as the Sparre Andersen result, and it describes the universal behavior of the free-space FPT statistics of one-dimensional Markov processes with symmetric steps [18,64,65]; thus, it even holds for Lévy flights with a diverging variance of the step length [65].

Depending on the initial distance to the target, there also exists an intermediate power-law regime corresponding to trajectories that make a brief excursion away from the target. The range of validity is strongly parameter and system dependent, but the resulting proximity effect is universal. We now address the aforementioned specific systems explicitly. The practical consequences of the proximity effect for gene regulation are discussed in the last subsection.

\section{A. Passive diffusion}

First, we focus on the unbiased diffusion of a particle in a hyperspherically symmetric domain of radius $R$ and a perfectly absorbing target with radius $r_{a}$ in the center. In our discussion, we also include the case of subdiffusion and consider the possibility of a fractal medium with fractal dimension $d_{f}[8,54,55]$. For this model, the qualitative regimes of the FPT behavior are captured by the single parameter $\nu=\frac{3}{2}-\left[\left(d_{f}+1\right) / d_{w}\right]$ [see Eqs. (9)-(11) as well as Ref. [48]]. If we limit ourselves to embedding dimensions $1 \leq d \leq 3$ and recall that $d_{w} \geq 2$ and $0 \leq d_{f} \leq d$, the possible values of $\nu$ fall in the interval spanned between three- and one-dimensional Brownian motion and hence $-\frac{1}{2} \leq \nu<\frac{3}{2}$. Moreover, the size $R$ of the confining domain factors out, as it merely sets the characteristic diffusion time $\tau=R^{d_{w}} / D_{d_{w}}$. Hence, without loss of generality, we introduce dimensionless units $x_{i}=\left[r_{i} / R\right]$, as well as the shorthand notation $\hat{x}=\left[2 / d_{w}\right] x$, and express time in units of $\tau$. The short-time asymptotics reads (for a derivation, see Ref. [48])

$$
\wp(t) \sim\left(\frac{x_{0}}{x_{a}}\right)^{\nu-1 / 2} \Phi\left(t ;\left|\hat{x}_{0}-\hat{x}_{a}\right|\right),
$$

which is valid for $t \lesssim \hat{x}_{a}^{2}, \hat{x}_{0}^{2}$. Apart from the $t$-independent prefactor, this result exhibits the Sparre Andersen form in Eq. (8) $[18,64]$. On the level of a stochastic differential equation, the effective process underlying the mean-field limit of diffusion in hyperspherically symmetric fractal media is indeed a one-dimensional Markov process in the radial coordinate (i.e., the squared Bessel process with parameter $\nu$ [62]). Yet, it is not symmetric because of the spurious geometric outward drift in the radial direction. This fact readily explains the additional dimensionalitydependent prefactor. Note that for $\nu>\frac{1}{2}$, the prefactor tends to be large in the limit $x_{a} \rightarrow 0$ and $x_{0} \not x_{a}$.
Following this short-time behavior, there is an intermediate regime for $[\Gamma(1+|\nu|) /|\Gamma(2-|\nu|)|]^{1 /(1-|\nu|)}\left(\hat{x}_{0} / 2\right)^{2} \ll$ $t \ll 1$ (Ref. [48]),

$$
\begin{aligned}
\wp(t) \sim & \left(\frac{x_{0}}{x_{a}}\right)^{2 \nu \Theta(-\nu)}\left[\left(\frac{\hat{x}_{0}}{2}\right)^{2|\nu|}-\left(\frac{\hat{x}_{a}}{2}\right)^{2|\nu|}\right] t^{-(1+\nu)} \\
& \times\left\{\frac{1}{\Gamma(|\nu|)}-\frac{\left(\hat{x}_{a} / 2\right)^{2|\nu|}}{\Gamma(-2|\nu|)}\left(\frac{\Gamma(1-|\nu|)}{\Gamma(1+|\nu|)}\right)^{2} t^{-\nu}\right\},
\end{aligned}
$$

for $\nu \neq 0$. Here, $\Theta(z)$ and $\Gamma(z)$ denote the Heaviside and Gamma functions. In the limit $\nu=0$ (including two-dimensional Brownian motion with $d_{f}=d_{w}=2$ ), we recover a logarithmic correction to the power-law scaling, $\wp(t) \sim 2 \log \left(x_{0} / x_{a}\right) /\left(t \log ^{2}(t)\right)$. Note that the leading terms of Eqs. (9) and (10) coincide for $|\nu|=$ $1 / 2$ (including one-dimensional Brownian motion with $d_{f}=1$ and $d_{w}=2$ ) in the regime $t \gg \hat{x}_{0}, \hat{x}_{a}$. Moreover, Eqs. (9) and (10) together describe direct FPT trajectories (Fig. 3), which do not touch the outer boundary of the system.

For any $\nu$, the long-time asymptotic corresponds exactly to the universal result in Eq. (6), with the coefficients $g^{(k)}$ and $h^{(k)}$ given in Eqs. (S27) and (S28) in Ref. [48], and it agrees perfectly with the simulation results in Fig. 2. As mentioned above, this generalizes and unifies the results in Ref. [8].

To explain the different limiting behavior of $g^{(k)}$ and $h^{(k)}$, we focus first on so-called noncompact dynamics, where the particle sparsely explores the space [8] and hence $d_{f}>d_{w}$ or $-\frac{1}{2} \leq \nu<0$. Examples include normal diffusion in $d \geq 2$ or diffusion and subdiffusion on fractal objects when the fractal dimension is larger than the walk dimension, $d_{f}>d_{w}$ [8]. Here, the aforementioned effective potential at the target site is the geometric or centrifugal bias away from the center controlled by the target radius $x_{a}$. From the exact solutions for $g^{(k)}$ and $h^{(k)}$ (given in Ref. [48]), it follows in the regime $d_{f}>d_{w}$ that, in the limit $x_{a} \rightarrow 0$, the correction term in Eqs. (S32) and (S33) vanishes, and we find, for arbitrary $x_{0}$,

$$
\begin{aligned}
\lambda_{0} & \sim \frac{4|\nu|(1+|\nu|)}{\hat{x}_{a}^{-2|\nu|}-|\nu|-1}, \\
\mathcal{C} & \sim \frac{4|\nu|(1+|\nu|)\left[\hat{x}_{a}^{-2|\nu|}-\hat{x}_{0}^{-2|\nu|}-|\nu| \hat{x}_{0}^{2}\right]}{\left(\hat{x}_{a}^{-2|\nu|}-|\nu|-1\right)^{2}} .
\end{aligned}
$$

As an important example, we consider the limit $\nu \rightarrow 0$ (i.e., null-recurrent dynamics [32]) and make use of the identity $\lim _{\delta \rightarrow 0}\left(1-x^{\delta}\right) / \delta=-\log (x)$. Thus, we smoothly recover the limiting case of critical compactness $(\nu=0)$,

$$
\lambda_{0} \sim \frac{2}{-\log \left(\hat{x}_{a}\right)}, \quad \mathcal{C} \sim \frac{\log \left(\frac{x_{0}}{x_{a}}\right)-\hat{x}_{0}^{2}}{\log ^{2}\left(\hat{x}_{a}\right)} .
$$


Equations (11) and (12) have the form of Eq. (7), and the prefactor in Eqs. (11) and (12) tends to be very small for $x_{0} \simeq x_{a}$. For example, in the case of normal diffusion in three dimensions $\left(d_{f}=3, d_{w}=2\right)$, we have $\left\langle T\left(x_{0}\right)\right\rangle=$ $\frac{1}{3}\left(x_{a}^{-1}-x_{0}^{-1}+\left(x_{a}^{2}-x_{0}^{2}\right) / 2\right)$ [18]. Thus, we find in the limit $x_{a} \rightarrow 0$ for $\nu=-\frac{1}{2}$ that $\mathcal{C}=\left\langle T\left(x_{0}\right)\right\rangle /\langle T(1)\rangle^{2}$ and $\lambda_{0}=\langle T(1)\rangle^{-1}$, as predicted by Eq. (7). Similarly, we find perfect agreement between Eq. (12) and the known result for two-dimensional diffusion (see, e.g., Ref. [19]). Other cases can be checked analogously by explicit computation.

In contrast, for $0 \leq \nu \leq \frac{3}{2}$, i.e., compact (recurrent) dynamics [32], where the particle densely explores the space (e.g., one-dimensional diffusion and diffusion on fractals when $d_{f}<d_{w}$ [8]), the Newton series in Eq. (2) does not converge with the first term in the limit $x_{a} \rightarrow 0$. Moreover, taking the limit $x_{a} \rightarrow 0$, we find that $\lambda_{0}$ becomes independent of $x_{a}$, and hence the notion of a target size effectively ceases to exist for $\nu<0$ such that taking the limit $x_{a}=\left(r_{a} / R\right) \rightarrow 0$ in this case corresponds to a change of the time unit; i.e., the result is invariant if we choose $\tau=\left(R-r_{a}\right)^{d_{w}} / D_{d_{w}}$.

Conversely, for nonrecurrent exploration $(\nu<0)$, there is no such invariance, and for any choice of $\tau$, the exponent $\lambda_{0}$ contains the term $x_{a}^{2|\nu|}$ [see Eq. (11)] such that $\lambda_{0}$ vanishes uniformly as $x_{a} \rightarrow 0$. This demonstrates that this limit for noncompact exploration corresponds mathematically to a genuine singular perturbation. The limits $r_{a} \rightarrow 0$ at fixed $R$ and $R \rightarrow \infty$ at fixed $r_{a}$, both giving $x_{a} \rightarrow 0$, are equivalent under an appropriate change of time units, and we already demonstrated that there is no difference between compact and noncompact FPT kinetics as long as $x_{a} \ll 1$. Taking the limit $x_{a} \rightarrow 0$ therefore has a very different mathematical meaning for $\nu \gtrless 0$. This is an important observation, which we will return to below.

\section{B. Deterministically biased diffusion}

Turning now from passive to biased (driven) diffusion, we first address Taylor dispersion, the one-dimensional diffusion under the influence of a constant bias $v$ away from an absorbing target at 0 , with a reflective boundary at $R$. Rescaling $x \rightarrow(x / R)$, the dimensionless Péclet number $\mathrm{Pe}=(v R / 2 D)$ captures all dynamical regimes [3]. The natural time unit is $\tau_{T}=\left(D / v^{2}\right)=(R / 2 v \mathrm{Pe})$, and the dynamics is nonrecurrent (noncompact). The short-time asymptotic reads (Ref. [48])

$$
\wp(t) \sim \Phi\left(t ; x_{0}\right) \mathrm{e}^{-\mathrm{Pe}\left(x_{0}+\mathrm{Pe} t\right)}=\left(2 \pi t^{3}\right)^{-1 / 2} \mathrm{e}^{-\left(x_{0}+2 \mathrm{Pe} t\right)^{2} / 4 t}
$$

for $t \ll 1$, which apparently deviates from the Sparre Andersen form [64]. Instead, in the limit of a large bias towards the target $\mathrm{Pe} \rightarrow-\infty$, the entire exponential term effectively acts as a cutoff at the deterministic FPT $t=x_{0} / 2 \mathrm{Pe}$. Note that a detailed analysis of the record and first-passage statistics of the corresponding discrete version of the model was studied in Ref. [66] using a random walk approach.

In the opposite limit when $t \rightarrow \infty$ and when $\mathrm{Pe} \gg 1$, the correction term given in Eq. (S37) vanishes and the Newton series in Eq. (2) reduces to (Ref. [48])

$$
\lambda_{0} \sim \mathrm{e}^{-2 \mathrm{Pe}}, \quad \mathcal{C} \sim \mathrm{e}^{-2 \mathrm{Pe}}\left(1-\mathrm{e}^{-2 \mathrm{Pe} x_{0}}\right) .
$$

This result has the universal form of Eq. (7). When $\mathrm{Pe} \rightarrow-\infty$, we need an increasing number of terms in Eqs. (6) and (2) as we approach the deterministic limit $\wp(t) \sim \delta\left(t-x_{0} / 2 \mathrm{Pe}\right)$. This finding agrees with the qualitative change of the behavior found in Ref. [66], when the external bias switches from strong repulsion to strong attraction.

Similar observations are made for two-dimensional diffusion under the influence of a radial bias $v(r)=$ $\left(v_{0} / r\right)$ [3,56] (Ref. [48]). We introduce $\mathrm{Pe}=\left(v_{0} / D\right)$ (Pe $>0$ for outward bias; note the different form with respect to the one-dimensional case) and dimensionless coordinates $x_{a, 0}=\left(r_{a, 0} / R\right)$. Because of the analogy to passive diffusion in a fractal medium when $\nu=-(\mathrm{Pe} / 2)$ (see also Refs. $[3,56]$ ), we immediately read off the shortand intermediate-time asymptotics in Eqs. (9) and (10). For $\mathrm{Pe} \rightarrow \infty$, the long-time behavior is given by Eq, (11), and we recover the universal form (7). As in the passive fractal case, the limit $\nu \rightarrow 0$ for $x_{a} \ll 1$ in Eq. (12) is approached symmetrically for $\mathrm{Pe} \rightarrow 0$ from below and above. For $\mathrm{Pe} \rightarrow-\infty$ (inward bias), increasingly many terms are needed in Eqs. (6) and (2) as we slowly approach the deterministic limit $\wp(t) \sim \delta\left(t-x_{0}^{2} / 2|\mathrm{Pe}|\right)$ (Ref. [48]).

As a second example, we address the FPT problem for diffusion in a harmonic potential, i.e., the OrnsteinUhlenbeck (OU) process or a concrete example for the Kramer's escape from a potential well [2,57-60]. Specifically, we consider a particle with mass $m$ and friction coefficient $\kappa$ starting at $x_{0}$ and diffusing in a harmonic potential $U(x)=m \omega^{2} x^{2} / 2$ with an absorbing boundary at $x_{a}$ (we choose $x_{0}<x_{a}$ ) in the overdamped limit $\kappa \ll \omega$. We introduce the characteristic length $l=$ $\sqrt{k_{B} T /\left(m \omega^{2}\right)}$ of the potential gauged by the thermal energy $k_{B} T$, rescale coordinates $x \rightarrow x / l$, and express time in natural units $\tau_{K}=\kappa /(m \omega)^{2}$. The OU process is positive recurrent [32] and hence corresponds to compact dynamics. When both $x_{a}$ and $x_{0}$ are not too large, the short-time asymptotics are obtained using the expansions in Ref. [67]. For $t \ll x_{a}^{-2}, x_{0}^{-2}$ (Ref. [48]),

$$
\begin{aligned}
& \wp(t) \sim \mathrm{e}^{\left[\left(x_{0}^{2}-x_{a}^{2}\right) / 4\right]} \Phi\left(t ; x_{a}-x_{0}\right) \\
& \circ \sum_{i=1}^{2} Q_{i}\left[\frac{1}{\sqrt{\pi t}}+\mathrm{e}^{P_{i}^{2} t} \operatorname{erfc}\left(P_{i} \sqrt{t}\right)\right],
\end{aligned}
$$


where $\circ$ denotes the one-sided convolution, $f(t) \circ g(t)=$ $\int_{0}^{t} f\left(t^{\prime}\right) g\left(t-t^{\prime}\right) d t^{\prime}$. Here, we use

$$
\begin{aligned}
Q_{1,2} & =\frac{x_{a}^{3}+x_{0}^{3}}{48} \mp \frac{1}{\sqrt{1+144 x_{a}^{-4}}}\left[\frac{3}{2}\left(\frac{1}{x_{a}}-\frac{x_{0}^{2}}{x_{a}^{3}}\right)+\frac{x_{a}^{3}+x_{0}^{3}}{48}\right], \\
P_{1,2} & =\frac{x_{a}^{3}}{48}\left(1 \pm \sqrt{1+144 x_{a}^{-4}}\right)
\end{aligned}
$$

where the upper sign is associated with the index 1 . The correction terms, which are necessary when $x_{a}$ and $x_{0}$ become significantly larger than 1 (yet remain moderate), extend the range of validity to longer $t$. In the case of $x_{a}$, $x_{0} \lesssim 1$, Eq. (15) reduces to

$$
\wp(t) \sim \mathrm{e}^{\left(x_{0}^{2}-x_{a}^{2}\right) / 4} \Phi\left(t ; x_{a}-x_{0}\right),
$$

which, apart from a $t$-independent prefactor, is the Sparre Andersen form [64].

For the OU process, the coefficients $h^{(k)}, g^{(k)}$ in Eqs. (2) and (6) are given in terms of $H_{0}^{n, 0}(x) \equiv$ $\left.(-1)^{n}\left(\partial^{n} / \partial \alpha^{n}\right) H_{\alpha}(x)\right|_{\alpha=0}$, where $H_{\alpha}(x)$ represents generalized Hermite functions, which are readily implemented, for instance, in Mathematica. For large barrier heights, the correction term in Eq. (S47) vanishes, and it can be shown (Ref. [48]) that the long-time FPT asymptotic attains the universal form of Eq. (7) with

$$
\begin{aligned}
\lambda_{0} & \sim \frac{x_{a}}{\sqrt{2 \pi}} \mathrm{e}^{-x_{a}^{2} / 2}, \\
\mathcal{C} & \sim \frac{x_{a}}{\sqrt{2 \pi}} \mathrm{e}^{-x_{a}^{2} / 2}\left[1-\frac{x_{a}}{\sqrt{2 \pi}} \mathrm{e}^{-x_{a}^{2} / 2} H_{0}^{1,0}\left(x_{0}\right)\right],
\end{aligned}
$$

and where we use the fact that

$H_{0}^{1,0}(x)=\frac{1}{2}\left[\pi \operatorname{erfi}\left(\frac{-x}{\sqrt{2}}\right)-x_{2}^{2} F_{2}\left(\begin{array}{cc}1, & 1 \\ 3 / 2, & 2\end{array} ;-\frac{x^{2}}{2}\right)-\delta\right]$.

Here, $\delta=\gamma+2 \log 2$ and $\gamma \simeq 0.5772$ is the EulerMascheroni constant [67], and we introduced the generalized hypergeometric function ${ }_{2} F_{2}(z)$ [67]. Note that since $\lim _{x \rightarrow-\infty} H_{0}^{1,0}(x) \sim-\log (x), \wp(t)$ only weakly depends on $x_{0}$ for large initial separations. Conversely, $\mathcal{C}$ can become very small in the case when $x_{0} \sim x_{a}$ : Developing an asymptotic expansion via analytic continuation to complex $x$ for Eq. (18) and for the exact mean FPT

$$
\left\langle T\left(x_{0}\right)\right\rangle=H_{0}^{1,0}\left(x_{0}\right)-H_{0}^{1,0}\left(x_{a}\right),
$$

we recover the universal form in Eq. (7). In the opposite case when $x_{a} \ll-1$, we approach the deterministic limit $\wp(t) \sim \delta\left(t-\log \left(x_{a} / x_{0}\right)\right)$ (see Refs. [48] and [58]).

\section{Discussion of the results in the context of molecular signaling in biological cells}

Nature apparently developed at least two distinct strategies to achieve both fast and precise molecular signaling in cells, necessary, for instance, in gene regulation. One is the energetically expensive and highly specific directed active transport by hitchhiking molecular motors, and it pertains to trafficking over large distances [26,68-71]. The second one is the spatial colocalization, i.e., proximity, of genes in which the protein encoded by one gene regulates the second gene $[15,21,31]$. The kinetics of this regulation process of the second gene include the diffusion of the protein of the first gene to the second gene. Despite the variety of motion patterns (diffusion, subdiffusion, diffusion on fractals, etc.) observed in different experiments [27,30,33,34], our results provide an explanation of the robustness of the proximity effect for efficient and precise transcription regulation.

Typically, in bacterial cells and eukaryotic nuclei, respectively, there are roughly $\lesssim 10^{2}[22,30]$ and $\lesssim 10^{4}$ [36] transcription factors (TFs) of one kind searching for their specific binding site on the cellular DNA. The linear extensions of bacteria and eukaryotic nuclei are $\sim 1 \mu \mathrm{m}$ and $\lesssim 5 \mu \mathrm{m}$, respectively [22,30,36,72]. The vast majority of the DNA binding sequences (promoters) are occupied by only a few proteins [45]. The promoter binding sites have a typical size of about $3 \mathrm{~nm}[22,30,36,72]$; thus, $x_{a} \simeq 10^{-3}$. The typical distance between colocalized genes is of order $\simeq 30-300 \mathrm{~nm}$ (the size of a transcription factory) $[31,36]$; hence, $\left|x_{0}-x_{a}\right| \simeq 5 \times 10^{-2}$.

Comparing $\wp(t)$ for different $\nu$ for these parameters on their respective natural time scales [Fig. 3(a)], we observe identical qualitative behavior, regardless of the compactness of the exploration. Namely, in all cases, we find a disparity of 2 to 4 orders of magnitude between the likelihood for direct trajectories-corresponding to Eqs. (9) and (10)—versus indirect trajectories-Eqs. (6), (11), and (12). This peak of accumulated probability mass is very narrow, i.e., 1 to 4 orders of magnitude narrower than the respective time scale $\lambda_{0}^{-1}$. The exponential long-time region is invariably statistically dominant [somewhat less so for compact dynamics, where the MFPT is shifted into the intermediate regime - compare the arrows in Fig. 3(a)] but has a $\gtrsim 4$ order-of-magnitude smaller value of $\wp(t)$. This means that, while the majority of trajectories are indirect and fall into the long-time regime, any two such indirect realizations will have massively different FPT (see also Refs. [25,73]). Conversely, direct trajectories are focused, i.e., have very similar FPT, giving rise to a sharp mode in the direct regime, $t_{\mathrm{typ}}=\left(x_{0}-x_{a}\right)^{2} / 6$. As it suffices that only a few of the $10^{2}-10^{4} \mathrm{TFs}$, i.e., the fastest $0.01 \%-1 \%$, actually need to bind to their target, the numerous, strongly dissimilar, indirect realizations are in fact irrelevant. Gene regulation kinetics, as uncovered by modern experimental techniques in single cells [23,74-77], therefore occur in the few-encounters limit, for which our results are relevant. 
Conversely, the results of Ref. [8] are relevant if we ask for the typical FPT of any of the TFs to their site, as would be measured in a bulk experiment for the behavior of an ensemble of genetically identical cells in a colony. In this sense, the results found here readily explain both the speed and the precision enhancement for the regulation of colocalized genes, irrespectively of the compactness of spatial exploration. The proof and explanation of the proximity effect in transcription regulation comprise our second main result. These principles can readily be extended to the more complex facilitated diffusion model $[15,21,78]$.

Further substantiating the robustness of the proximity effect is the fact that the results qualitatively remain unchanged in the presence of a bias [Fig. 3(b)]. Naturally, a bias towards the target shifts the long-time regime towards shorter $t$. It might appear somewhat surprising at first that the bias does not affect, apart from a trivial renormalization, the statistics, i.e., the width and position of direct trajectories [Fig. 3(b)]. This observation is, however, a straightforward consequence of the independence of drift and diffusion. It highlights the intrinsic universality of direct paths and conceptually extends the Sparre Andersen universality [18]. Remarkably, proximity effects persist in the OU process [Fig. 3(b), violet], for which direct, strictly uphill trajectories are typical and in turn imply excessive fluctuations of FPT times of indirect trajectories.

\section{CONCLUDING REMARKS}

Our rigorous results provide a general method to determine the exact long-time behavior of a smooth onesided probability density with finite moments from its Laplace transform. They extend the existing generic asymptotic inversion methods, such as Tauberian theorems for slowly varying functions, to functions varying exponentially fast at long times. Here, this new method enabled us to unify the first-passage time statistics of all FPT densities with asymptotic exponential decay, both passive and those under the influence of a deterministic bias, into a single universality class. Moreover, they provided a deep physical insight into the general effects of (non)recurrent spatial exploration on the FPT behavior and highlighted the qualitatively very different meaning of assuming a large volume or a small target size. Beyond the theoretical advance, our results are directly relevant in a biophysical context.

Thus, modern experimental methods allow the observation of individual molecular regulation events. It is therefore timely to extend the classical vista of mean-rate approaches to biochemical kinetics and consider the full distribution of FPTs $[79,80]$. The theoretical results presented here will be a quantitative basis for the development and analysis of massive single-molecule experiments for FPT dynamics in living cells or chemical reactions in micro- and nano-containers. The few-encounter limit introduced here, together with the proximity effect, complements the many-encounter regime associated with the mean FPT theory developed in Ref. [8]. In this latter regime, we fully corroborate the results of Ref. [8], in particular, the observation that the initial distance to the target will be critically more important for compact exploration.

Our results have immediate consequences for the interpretation of single-molecule reaction or binding experiments (e.g., Ref. [20] and references therein) that nowadays allow one to directly visualize the few-encounter regime. Namely, the quantification of reaction kinetics is paradigmatically reduced to the mean FPT $[2,7,47]$, which is meaningful if we are interested in systems in which most of the molecules are required to react. In contrast, when only a small number of reactive encounters are required, e.g., the binding of a few TFs to their targets deciding the regulatory pathway of a cell [23] or phosphorylation cascades in cellular signal transduction propagating towards the nucleus [81], our results show that typical and direct realizations are essential. In this few-encounter regime, mean FPT-based concepts grossly underestimate the speed and precision of experimentally observed signaling kinetics and would thus lead to severe parameter misjudgments. These would deteriorate further for FPT cascades. In other words, the notion of a kinetic rate in the traditional bulk sense ceases to exist in the few-encounter regime. The universality of the proximity effect in target search kinetics enabling temporal signal focusing therefore challenges traditional views on biochemical reactions in cells and provides the basis for new models for molecular regulatory kinetics in the few-encounter regime.

\section{ACKNOWLEDGMENTS}

We thank Olivier Bénichou for instigating the present work and for discussions. A. G. acknowledges funding through an Alexander von Humboldt grant and ARRS Project No. Z1-7296.

[1] M. von Smoluchowski, Drei Vorträge über Diffusion, Brownsche Molekularbewegung und Koagulation von Kolloidteilchen, Phys. Z. 17, 557 (1916).

[2] P. Hänggi, P. Talkner, and M. Borkovec, Reaction-Rate Theory: Fifty Years after Kramers, Rev. Mod. Phys. 62, 251 (1990).

[3] S. Redner, A Guide to First Passage Processes. (Cambridge University Press, New York, 2001).

[4] M. Shlesinger, Mathematical Physics: Search Research, Nature (London) 443, 281 (2006).

[5] First-Passage Phenomena and Their Applications, edited by R. Metzler, G. Oshanin, and S. Redner (World Scientific, Singapore, 2014).

[6] R. Kopelman, Fractal Reaction Kinetics, Science 241, 1620 (1988). 
[7] A. Szabo, K. Schulten, and Z. Schulten, First Passage Time Approach to Diffusion Controlled Reactions, J. Chem. Phys. 72, 4350 (1980).

[8] O. Bénichou, C. Chevalier, J. Klafter, B. Meyer, and R. Voituriez, Geometry-Controlled Kinetics, Nat. Chem. 2, 472 (2010).

[9] Z. Schuss, Theory and Applications of Stochastic Processes: An Analytical Approach (Springer, New York, 2010).

[10] E. Ben-Naim, S. Redner, and F. Leyvraz, Decay Kinetics of Ballistic Annihilation, Phys. Rev. Lett. 70, 1890 (1993).

[11] G. Oshanin, A. Stemmer, S. Luding, and A. Blumen, Smoluchowski Approach for Three-Body Reactions in One Dimension, Phys. Rev. E 52, 5800 (1995).

[12] B. Meyer, C. Chevalier, R. Voituriez, and O. Bénichou, Universality Classes of First-Passage-Time Distribution in Confined Media, Phys. Rev. E 83, 051116 (2011).

[13] O. Bénichou and R. Voituriez, From First-Passage Times of Random Walks in Confinement to Geometry-Controlled Kinetics, Phys. Rep. 539, 225 (2014).

[14] Z. Schuss, A. Singer, and D. Holcman, The Narrow Escape Problem for Diffusion in Cellular Microdomains, Proc. Natl. Acad. Sci. U.S.A. 104, 16098 (2007).

[15] O. Pulkkinen and R. Metzler, Distance Matters: The Impact of Gene Proximity in Bacterial Gene Regulation, Phys. Rev. Lett. 110, 198101 (2013).

[16] Z. Wunderlich and L. A. Mirny, Spatial Effects on the Speed and Reliability of Protein-DNA Search, Nucleic Acids Res. 36, 3570 (2008).

[17] D. Holcman and Z. Schuss, Time Scale of Diffusion in Molecular and Cellular Biology, J. Phys. A 47, 173001 (2014).

[18] A. Godec and R. Metzler, First Passage Time Distribution in Heterogeneity Controlled Kinetics: Going Beyond the Mean First Passage Time, Sci. Rep. 6, 20349 (2016).

[19] A. Godec and R. Metzler, Optimization and Universality of Brownian Search in a Basic Model of Quenched Heterogeneous Media, Phys. Rev. E 91, 052134 (2015).

[20] M. Kurzynski, K. Palacz, and P. Chelminiak, Time Course of Reactions Controlled and Gated by Intramolecular Dynamics of Proteins: Predictions of the Model of Random Walk on Fractal Lattices, Proc. Natl. Acad. Sci. U.S.A. 95, 11685 (1998).

[21] G. Kolesov, Z. Wunderlich, O. N. Laikova, M. S. Gelfand, and L. A. Mirny, How Gene Order Is Influenced by the Biophysics of Transcription Regulation, Proc. Natl. Acad. Sci. U.S.A. 104, 13948 (2007).

[22] W. Bialek and S. Setayeshgar, Physical Limits to Biochemical Signaling, Proc. Natl. Acad. Sci. U.S.A. 102, 10040 (2005).

[23] G. W. Li and X.S. Xie, Central Dogma at the SingleMolecule Level in Living Cells, Nature (London) 475, 308 (2011).

[24] J. C. M. Gebhardt, D. M Suter, R. Roy, Z.W Zhao, A. R. Chapman, S. Basu, T. Maniatis, and X. S. Xie, Single-Molecule Imaging of Transcription Factor Binding to DNA in Live Mammalian Cells, Nat. Methods 10, 421 (2013).

[25] C. Mejía-Monasterio, G. Oshanin, and G. Schehr, First Passages for a Search by a Swarm of Independent Random Searchers, J. Stat. Mech. (2011) P06022.
[26] A. Godec and R. Metzler, Signal Focusing through Active Transport, Phys. Rev. E 92, 010701(R) (2015).

[27] C. Di Rienzo, V. Piazza, E. Gratton, F. Beltram, and F. Cardarelli, Probing Short-Range Protein Brownian Motion in the Cytoplasm of Living Cells, Nat. Commun. 5, 5891 (2014).

[28] S. A. Gorski, M. Dundr, and T. Misteli, The Road Much Traveled: Trafficking in the Cell Nucleus, Curr. Opin. Cell Biol. 18, 284 (2006).

[29] F. Höfling and T. Franosch, Anomalous Transport in the Crowded World of Biological Cells, Rep. Prog. Phys. 76, 046602 (2013).

[30] W. Bialek, Biophysics: Searching for Principles (Princeton University Press, New Jersey, 2012).

[31] P. Fraser and W. Bickmore, Nuclear Organization of the Genome and the Potential for Gene Regulation, Nature (London) 447, 413 (2007).

[32] J. J. Liou, Recurrence and Transience of Gaussian Diffusion Processes, Kodai Math. J. 13, 210 (1990).

[33] A. Bancaud, S. Huet, N. Daigle, J. Mozziconacci, J. Beaudouin, and J. Ellenberg, Molecular crowding affects diffusion and binding of nuclear proteins in heterochromatin and reveals the fractal organization of chromatin, EMBO J. 28, 3785 (2009).

[34] C. Lanctót, T. Cheutin, M. Cremer, G. Cavalli, and T. Cremer, Dynamic Genome Architecture in the Nuclear Space: Regulation of Gene Expression in Three Dimensions, Nat. Rev. Genet. 8, 104 (2007).

[35] E. Toro and L. Shapiro, Bacterial Chromosome Organization and Segregation, Cold Spring Harbor Persp. Biol. 2, a000349 (2010).

[36] J. Zlatanova and K. E. van Holde, Molecular Biology: Structure and Dynamics of Genomes and Proteomes (Garland Science, New York, 2015).

[37] O. Bénichou, C. Loverdo, M. Moreau, and R. Voituriez, Intermittent Search Strategies, Rev. Mod. Phys. 83, 81 (2011).

[38] G. E. Viswanathan, M. G. E. da Luz, E. P. Raposo, and H. E. Stanley, The Physics of Foraging: An Introduction to Random Searches and Biological Encounters (Cambridge University Press, New York, 2011).

[39] A. L. Lloyd and R. M. May, How Viruses Spread Among Computers and People, Science 292, 1316 (2001).

[40] G. M. Viswanathan, S. V. Buldyrev, S. Havlin, M. G. E. da Luz, E. P. Raposo, and H. Eugene Stanley, Optimizing the Success of Random Searches, Nature (London) 401, 911 (1999).

[41] V. V. Playulin, A. V. Chechkin, and R. Metzler, Lévy Flights Do Not Always Optimize Random Blind Search for Sparse Targets, Proc. Natl. Acad. Sci. U.S.A. 111, 2931 (2014).

[42] D. W. Sims, M. J. Witt, A. J. Richardson, E. J. Southall, and J. D. Metcalfe, Encounter Success of Free-Ranging Marine Predator Movements Across a Dynamic Prey Landscape, Proc. Biol. Sci. 273, 1195 (2006).

[43] J. D. Houghton, T. K. Doyle, M. W. Wilson, J. Davenport, and G. C. Hays, Jellyfish Aggregations and Leatherback Turtle Foraging Patterns in a Temperate Coastal Environment, Ecology 87, 1967 (2006).

[44] C. J. A. Bradshaw, M. A. Hindell, M. D. Sumner, and K. J. Michael, Loyalty Pays: Potential Life History 
Consequences of Fidelity to Marine Foraging Regions by Southern Elephant Seals, Animal Behaviour 68, 1349 (2004).

[45] C. T. Harbison et al., Transcriptional Regulatory Code of a Eukaryotic Genome, Nature (London) 431, 99 (2004).

[46] S. Redner and B. Meerson, First Invader Dynamics in Diffusion-Controlled Absorption, J. Stat. Mech. (2014) P06019.

[47] K. Lindenberg, V. Seshadri, K. E. Shuler, and G. H. Weiss, Lattice Random Walks for Sets of Random Walkers. First Passage Times, J. Stat. Phys. 23, 11 (1980).

[48] See Supplemental Material at http://link.aps.org/ supplemental/10.1103/PhysRevX.6.041037 for detailed proofs of the general results (2)-(6) as well as for explicit calculations and exact results for all the model systems considered in the main text.

[49] W. Feller, An Introduction to Probability Theory and Its Applications (Wiley, New York, 1968), Vol. II.

[50] C. W. Gardiner, Handbook of Stochastic Methods for Physics, Chemistry and the Natural Sciences (Springer, Berlin-Heidelberg, 1985).

[51] A. Godec and R. Metzler, Linear Response, FluctuationDissipation, and Finite-System-Size Effects in Superdiffusion, Phys. Rev. E 88, 012116 (2013).

[52] T. Guérin, N. Levernier, O. Bénichou, and R. Voituriez, Mean First-Passage Times of Non-Markovian Random Walkers in Confinement, Nature (London) 534, 356 (2016).

[53] T. Verechtchaguina, I. M. Sokolov, and L. SchimanskyGeier, First Passage Time Densities in Non-Markovian Models with Subthreshold Oscillations, Europhys. Lett. 73, 691 (2006).

[54] B. O'Shaughnessy and I. Procaccia, Analytical Solutions for Diffusion on Fractal Objects, Phys. Rev. Lett. 54, 455 (1985).

[55] D. ben-Avraham and S. Havlin, Diffusion and Reactions in Fractals and Disordered Systems (Cambridge University Press, Cambridge, England, 2002).

[56] J. Koplik, S. Redner, and E. J. Hinch, Universal and Nonuniversal First-Passage Properties of Planar Multipole Flows, Phys. Rev. Lett. 74, 82 (1995).

[57] H. A. Kramers, Brownian Motion in a Field of Force and the Diffusion Model of Chemical Reactions, Physica (Amsterdam) 7, 284 (1940).

[58] A. J. F. Siegert, On the First Passage Time Probability Problem, Phys. Rev. 81, 617 (1951).

[59] K. Schulten, Z. Schulten, and A. Szabo, Dynamics of Reactions Involving Diffusive Barrier Crossing, J. Chem. Phys. 74, 4426 (1981).

[60] G. Margolin and E. Barkai, Single-Molecule Chemical Reactions: Reexamination of the Kramers Approach, Phys. Rev. E 72, 025101 (2005).

[61] D. Grebenkov, First Exit Times of Harmonically Trapped Particles: A Didactic Review, J. Phys. A 48, 013001 (2015).

[62] D. Revuz and M. Yor, Continuous Martingales and Brownian Motion (Springer-Verlag, Berlin-Heidelberg, 1999).

[63] D. W. Stroock and S. R.S. Varadhan, Multidimensional Diffusion Processes (Springer, Berlin-Heidelberg, 2006).
[64] E. Sparre Andersen, On the Fluctuations of Sums of Random Variables, Math. Scand. 1, 263 (1953); On the Fluctuations of Sums of Random Variables. II., Math. Scand. 2, 195 (1954).

[65] T. Koren, M. A. Lomholt, A. V. Chechkin, J. Klafter, and R. Metzler, Leapover Lengths and First Passage Time Statistics for Lévy Flights, Phys. Rev. Lett. 99, 160602 (2007).

[66] S. N. Majumdar, G. Schehr, and G. Wergen, Record Statistics and Persistence for a Random Walk with a Drift, J. Phys. A 45, 355002 (2012).

[67] M. Abramowitz and I. A. Stegun, Handbook of Mathematical Functions (Dover, New York, 1970).

[68] B. Alberts et al., Molecular Biology of the Cell (Garland, New York, NY, 2002).

[69] O. Bénichou, C. Loverdo, M. Moreau, and R. Voituriez, Enhanced Reaction Kinetics in Biological Cells, Nat. Phys. 9, 1342008.

[70] K. Chen, B. Wang, and S. Granick, Memoryless SelfReinforcing Directionality in Endosomal Active Transport within Living Cells, Nat. Mater. 14, 589 (2015).

[71] A. Godec and R. Metzler, Active Transport Improves the Precision of Linear Long Distance Molecular Signalling, J. Phys. A 49, 364001 (2016).

[72] T. Cavalier-Smith, Nuclear Volume Control by Nucleoskeletal DNA, Selection for Cell Volume and Cell Growth Rate, and the Solution of the DNA C-Value Paradox, J. Cell Sci. 34, 247 (1987).

[73] T. G. Mattos, C. Mejìa-Monasterio, R. Metzler, and G. Oshanin, First Passages in Bounded Domains: When Is the Mean First Passage Time Meaningful?, Phys. Rev. E 86, 031143 (2012).

[74] J. Yu, J. Xiao, X. Ren, K. Lao, and X. S. Xie, Probing Gene Expression in Live Cells, One Protein Molecule at a Time, Science 311, 1600 (2006).

[75] L. Cai, N. Friedman, and X. S. Xie, Stochastic Protein Expression in Individual Cells at the Single Molecule Level, Nature (London) 440, 358 (2006).

[76] A. Honigmann, V. Mueller, H. Ta, A. Schoenle, E. Sezgin, S. W. Hell, and C. Eggeling, Scanning STED-FCS Reveals Spatiotemporal Heterogeneity of Lipid Interaction in the Plasma Membrane of Living Cells, Nat. Commun. 5, 5412 (2014).

[77] A. V. Weigel, M. M. Tamkun, and D. Krapf, Quantifying the Dynamic Interactions Between a Clathrin-Coated Pit and Cargo Molecules, Proc. Natl. Acad. Sci. U.S.A. 110, E4591 (2013).

[78] P. H. Hippel and O. G. Berg, Facilitated Target Location in Biological Systems, J. Biol. Chem. 264, 675 (1989).

[79] A. Mahmutovic, D. Fange, O. Berg, and J. Elf, Lost in Presumption: Stochastic Reactions in Spatial Models, Nat. Methods 9, 1163 (2012).

[80] C. Di Rienzo, E. Gratton, F. Beltram, and F. Cardarelli, Spatiotemporal Fluctuation Analysis: A Powerful Tool for the Future Nanoscopy of Molecular Processes, Biophys. J. 111, 679 (2016).

[81] T. Lu, T. Shen, C. Zhong, J. Hastym, and P. Wolynes, Statistics of Cellular Signal Transduction as a Race to the Nucleus by Multiple Random Walkers in Compartment/ Phosphorylation Space, Proc. Natl. Acad. Sci. U.S.A. 103, 16752 (2006). 